\title{
Éditorial
}

\section{Actualité de Paul-Claude Racamier}

\section{A journey with Paul-Claude Racamier}

\author{
Jean Marc GUILÉ \\ Rédacteur en chef
}

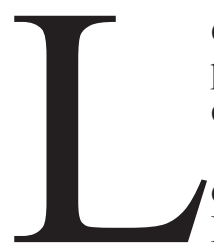

e travail clinique et théorique du psychiatre et psychanalyste français Paul-Claude Racamier (19241996), cofondateur du Collège de Psychanalyse Groupale et Familiale et ancien directeur de l'Institut de psychanalyse de Paris, se révèle d'une éclairante actualité. Ce numéro de Perspectives Psy donne la voix à ceux qui ont travaillé avec lui, ont poursuivi son œuvre auprès des patients et de leurs familles, tout particulièrement au Centre psychothérapeutique de La Velotte, et, avec Pascale de Sainte Marie et Geneviève Veuriot, ont organisé le colloque «Originaire et psychose » en 2011 à Besançon.

Racamier a renouvelé le regard clinique sur les pathologies prégénitales qui s'expriment par une déstructuration psychique profonde comme la psychose ou bien dans l'agir et l'emprise sur l'autre comme les troubles de la personnalité et les organisations perverses. Explorant l'ontogénie de la pensée et du lien humain, il a mis en lumière le rôle fondamental du deuil comme organisateur psychique. En dialogue avec d'autres explorateurs de l'archaïque comme Klein, Winnicott et Mahler, il met de l'avant le deuil originaire, mouvement à la fois actif et passif, que le bébé opère lorsqu'il abandonne l'illusion de fusion toute-puissante avec la figure maternelle. " C'est à partir du deuil que se découvre l'objet 》 (Guillaumin et Racamier, 1994, p. 1166). Au moment où dans le développement psychique de l'enfant se distinguent objet externe et objet interne, se crée une séparation entre le dedans et le dehors, entre le réel et l'imaginaire. Ce mouvement est le creuset d'un nouvel équilibre des investissements objectaux et narcissiques au sein duquel le sujet, renonçant à la totale maitrise de l'objet d'amour primaire, se découvre progressivement luimême comme sujet.

Le travail de Paul-Claude Racamier est prodigieusement actuel pour les soignants qui accompagnent les patients aux prises avec des pathologies de l'agir ou des conduites suicidaires vis-à-vis desquelles le travail de séparation psychique est l'enjeu (Mille, 2011). Les élaborations théoriques développées dans les deux dossiers de ce numéro prennent un relief grandissant sur la toile de fond des évolutions sociétales actuelles où se remodèlent les questions des différences de générations et de sexes. L'un des défis réside dans ce qu'il advient de la place imaginaire de la figure paternelle. Sommes-nous face à une évolution 
vers un tiers séparateur non-sexué ? Plus encore, la riche notion apportée par Racamier d'antœdipe, fonctionnant comme métaphore du fantasme d'auto-engendrement, nous offre une clé de lecture supplémentaire des mouvements psychiques individuels et groupaux actuels.

Dans les situations cliniques et institutionnelles où les deuils sont avortés-nés, et expulsés du psychisme pour reprendre le concept de Racamier, pas de vraie séparation, ni de castration. On se place dans l'incestuel (Racamier, 1998), c'est-à-dire dans les agirs et

\section{RÉFÉRENCES BIBLIOGRAPHIQUES}

1. De Sainte Marie P. (2012). Originaire et psychose. Perspectives Psy, 51 (3) : 215-218.

2. Guillaumin J., Racamier P.-C. (1994). Entretien de Jean Guillaumin avec Paul-Claude Racamier à propos de son dernier livre. Revue Française de Psychanalyse, 4 : 1166-1176. les impasses de la symbolisation où la pensée s'arrête et la créativité s'éteint.

Le premier dossier expose le travail du deuil sur les différentes scènes où il se déploie ; le second dossier et le texte de Libre cours illustrent le travail clinique et institutionnel auprès des patients et des équipes. Un travail tout en demi-teintes où se cultive une ambiguïté tempérée au profit du plaisir de penser et de la créativité du soin. Comme l'exprime bien Pascale de Sainte-Marie, il s'agit de proposer aux patients « des réponses ambiguës aux vécus paradoxaux » (2012, p. 215).

\section{Communiqué}

\section{COPELFI}

(Association de conférences de psychiatrie de l'enfant et de l'adolescent de langue française en Israël)

et

Le groupe d'études psychologiques et psychiatriques de langue française en Israël

Avec le parrainage de

La Société française de psychiatrie de l'enfant et de

l'adolescent et disciplines associées

L'Euvre de secours aux enfants (OSE)

La société israélienne de psychiatrie de l'enfant et de l'adolescent

La société israélienne de psychanalyse de l'enfant et de l'adolescent

\section{Séparer un enfant : la souffrance et la nécessité Le placement et ses alternatives}

\section{Conférence de psychiatrie de l'enfant et de l'adolescent de langue française en Israël}

28 octobre - 4 novembre 2012

Tel-Aviv/Jérusalem/Beer Sheva/Eilat

\section{Renseignements}

Fabienne Bensimon, COPELFI, 34 allée Gambetta, 93250 Villemomble, France

Tél : 0148543997

E-mail : ass.copelfi@club-internet.fr

Site : www.copelfi.fr 